\title{
The follow-up European VLBI Network observations of twelve GPS radio sources at $5 \mathrm{GHz}$
}

\author{
L. Cui ${ }^{1,2}$, X. Liu ${ }^{1}$, J. Liu ${ }^{1,2}$, H.-G. Song ${ }^{1,2}$, and Z. Ding ${ }^{1,2}$ \\ 1 Urumqi Observatory, the National Astronomical Observatories, CAS, 40-5 South Beijing Road, Urumqi 830011, P.R. China \\ e-mail: liux@uao.ac.cn \\ 2 Graduate University of the Chinese Academy of Sciences, Beijing 100049, P.R. China
}

Received 15 January 2010 / Accepted 26 April 2010

\begin{abstract}
Aims. Gigahertz peaked spectrum (GPS) radio sources are compact extragalactic radio sources, assumed to be young radio-loud active galactic nuclei and ideal objects for studying the early evolution of extragalactic radio sources. The Very Long Baseline Interferometry (VLBI) observation with high resolution is vital for studying the structure of these compact sources.

Methods. We defined a sub-sample of twelve GPS sources which have not been observed with the VLBI before, from the Parkes half-Jansky sample, and carried out VLBI observations at $1.6 \mathrm{GHz}$ and $5 \mathrm{GHz}$ with the European VLBI Network (EVN) in 2006 and 2008, respectively, to classify the source structure and to find compact symmetric objects (CSOs). Additionally, we carried out the $4.85 \mathrm{GHz}$ flux density observations for these sources with the Urumqi 25-m telescope between the years 2007 and 2009 to study whether there is any variability in the total flux density of the GPS sources.

Results. The results of the $5 \mathrm{GHz}$ VLBI observations and total flux densities of these sources are presented in this paper. From the VLBI morphologies, the spectral indices of components and the total flux variability of the twelve targets, we firmly classify three sources J0210+0419, J1135-0021, and J2058+0540 as CSOs, and classify J1057+0012, J1203+0414, and J1600-0037 as corejet sources. The others J0323+0534, J0433-0229, J0913+1454, J1109+1043, and J1352+0232 are labelled CSO candidates, and $\mathrm{J} 1352+1107$ is a complex feature. Apart from core-jet sources, the total flux densities of the CSOs and candidates are quite stable at $5 \mathrm{GHz}$ both during a long-term of $\sim 20$ years relative to the PKS90 data and in a period between 2007 and 2009. The total flux densities are resolved-out by more than $20 \%$ in the $5 \mathrm{GHz}$ VLBI images for 6 sources, probably because of diffuse emission. In addition, we estimated the jet viewing angles $(\Theta)$ for the confirmed CSOs by using the double-lobe flux ratio of the sources, the result being indicative of relatively large $\Theta$ for the CSOs.
\end{abstract}

Key words. galaxies: nuclei - quasars: general - radio continuum: galaxies - galaxies: active - galaxies: jets

\section{Introduction}

Gigahertz peaked spectrum (GPS) radio sources are powerful $\left(P_{1.4 \mathrm{GHz}} \geq 10^{25} \mathrm{~W} \mathrm{~Hz}^{-1}\right)$ and compact extragalactic radio sources characterized by a convex radio spectrum peaking at between 0.3 and a few $\mathrm{GHz}$ (see O'Dea 1998, for a review). Sources that peak at $>5 \mathrm{GHz}$ are called high frequency peakers (HFP, Dallacasa et al. 2000), which are understood to be even more compact and younger. The spectrum turnover of the GPS sources is caused by synchrotron self-absorption (de Vries et al. 2009), in general, and it is also possible that free-free absorption plays a role in producing the spectral turnover in some cases (e.g., Luo et al. 2007; Orienti \& Dallacasa 2008).

Extended emission in GPS source is uncommon especially for GPS galaxies, most of which are truly compact $\left(<1 \mathrm{kpc} h^{-1}\right)$ (Stanghellini et al. 2005). The compactness of GPS sources is most likely due to their youth $\left(<10^{4}\right.$ years $)$ according to a spectral ageing analysis (Murgia et al. 2003). The youth scenario is supported by the hotspot kinematics in a couple of GPS sources that are also identified as compact symmetric objects (CSOs) (Giroletti \& Polatidis 2009). A unification model assumes that GPSs/CSOs will evolve into compact steep spectrum (CSS) sources or medium-sized symmetric objects (MSOs, $\left.1-20 \mathrm{kpc} h^{-1}\right)$, which in turn, evolve into large radio galaxies ( $>20 \mathrm{kpc} h^{-1}$ ), i.e., FRI/II radio sources (Fanti et al. 1995, 2009; Snellen et al. 2000).
Furthermore, the GPS sources are associated with either galaxies or quasars. The GPS quasars usually exhibit some form of core-jet structure, while the GPS galaxies often show CSOlike or compact doubles (CD) (e.g., Orienti et al. 2006; Liu et al. 2007), suggesting that the GPS galaxies have relatively large jetviewing angles. The GPS galaxies have very low polarization (less than $0.5 \%$ at $5 \mathrm{GHz}$, Xiang et al. 2006), which is probably because of the large Faraday depth in the line of sight, implying that their host AGN are largely edge-on to us.

Complete samples of GPS sources are statistically vital for studying the early evolution of radio-loud AGN and the triggering of their nuclear activity, such as the 1 Jy complete GPS sample in the northern sky (Stanghellini et al. 1998), the Parkes half-Jansky (PHJ) sample in the southern/equatorial sky (Snellen et al. 2002), and the CORALZ sample at low redshift (Snellen et al. 2004). The Very Long Baseline Interferometry (VLBI) technique is a key tool for studying the morphologies of GPS sources from the GPS samples. We defined a subsample of twelve GPS sources with declination $>-5^{\circ}$ that had not been observed with the VLBI before, from the PHJ sample, and carried out the VLBI observations at $1.6 \mathrm{GHz}$ and $5 \mathrm{GHz}$ of them in 2006 and 2008, respectively. It is found that most of the sources exhibit compact double structure by analysing the $1.6 \mathrm{GHz}$ European VLBI Network (EVN) observation (Liu et al. 2007, hereafter Paper I). In the follow-up $5 \mathrm{GHz}$ EVN observation, we demonstrate that we can resolve their VLBI structure more 
Table 1. The GPS sample.

\begin{tabular}{|c|c|c|c|c|c|c|c|c|c|c|c|c|}
\hline $\begin{array}{c}1 \\
\text { IAU } \\
\text { name }\end{array}$ & $\begin{array}{c}2 \\
\text { Other }\end{array}$ & $\begin{array}{c}3 \\
\text { Id }\end{array}$ & $\begin{array}{l}4 \\
z\end{array}$ & $\begin{array}{c}5 \\
\text { Scale } \\
\text { (pc/mas) }\end{array}$ & $\begin{array}{c}6 \\
\theta_{5 \mathrm{GHz}} \\
\text { (mas) }\end{array}$ & $\begin{array}{c}7 \\
\mathrm{LS} \\
(\mathrm{pc})\end{array}$ & $\begin{array}{c}8 \\
S_{4.85 U r} \\
(\mathrm{mJy})\end{array}$ & $\begin{array}{c}9 \\
\text { Resolved } \\
(\%)\end{array}$ & $\begin{array}{c}10 \\
\text { Comp. }\end{array}$ & $\begin{array}{c}11 \\
S_{\text {int }}^{1.65} \\
(\mathrm{mJy})\end{array}$ & $\begin{array}{c}12 \\
S_{\mathrm{int}}^{5} \\
(\mathrm{mJy})\end{array}$ & $\begin{array}{c}13 \\
\alpha_{1.65}^{5}\end{array}$ \\
\hline J0210+0419 & B0208+040 & G & $1.5^{*}$ & $6.11 h^{-1}$ & 84 & $513 h^{-1}$ & $282 \pm 2$ & $\sim 14$ & $\begin{array}{l}\text { A } \\
\text { B }\end{array}$ & $\begin{array}{l}375 \\
205\end{array}$ & $\begin{array}{c}144 \\
99\end{array}$ & $\begin{array}{l}0.86 \\
0.66\end{array}$ \\
\hline J0323+0534 & $4 C+05.14$ & G & 0.1785 & $2.13 h^{-1}$ & 48 & $102 h^{-1}$ & $796 \pm 21$ & $\sim 83$ & $\begin{array}{l}\mathrm{A} \\
\mathrm{B}\end{array}$ & $\begin{array}{l}1270 \\
548\end{array}$ & $\begin{array}{c}102 \\
-\end{array}$ & $\begin{array}{c}2.27 \\
-\end{array}$ \\
\hline J0433-0229 & $4 C-02.17$ & G & 0.53 & $4.47 h^{-1}$ & 72 & $322 h^{-1}$ & $612 \pm 5$ & $\sim 27$ & $\begin{array}{l}\text { A } \\
\text { B }\end{array}$ & $\begin{array}{c}1045 \\
111\end{array}$ & $\begin{array}{c}396 \\
16\end{array}$ & $\begin{array}{l}0.88 \\
1.75\end{array}$ \\
\hline J0913+1454 & B0910+151 & G & $0.47 *$ & $4.19 h^{-1}$ & 76 & $318 h^{-1}$ & $275 \pm 18$ & $\sim 8$ & $\begin{array}{l}\mathrm{A} \\
\mathrm{B}\end{array}$ & $\begin{array}{l}501 \\
157\end{array}$ & $\begin{array}{c}208 \\
45\end{array}$ & $\begin{array}{l}0.79 \\
1.13\end{array}$ \\
\hline $\mathrm{J} 1057+0012$ & B1054+004 & G & $0.65^{*}$ & $4.97 h^{-1}$ & 13 & $65 h^{-1}$ & $355 \pm 21$ & $\sim 11$ & $\begin{array}{l}\mathrm{A} \\
\mathrm{B}\end{array}$ & $\begin{array}{c}550 \\
58\end{array}$ & $\begin{array}{c}299 \\
-\end{array}$ & $\begin{array}{c}0.55 \\
-\end{array}$ \\
\hline $\mathrm{J} 1109+1043$ & B1107+109 & G & $0.55^{*}$ & $4.62 h^{-1}$ & 63 & $290 h^{-1}$ & $420 \pm 7$ & $\sim 8$ & $\begin{array}{l}\text { A } \\
\text { B }\end{array}$ & $\begin{array}{l}984 \\
311\end{array}$ & $\begin{array}{c}281 \\
91\end{array}$ & $\begin{array}{l}1.13 \\
1.11\end{array}$ \\
\hline J1135-0021 & $4 C-00.45$ & G & 0.975 & $5.75 h^{-1}$ & 110 & $633 h^{-1}$ & $422 \pm 13$ & $\sim 15$ & $\begin{array}{l}\text { A } \\
\text { B }\end{array}$ & $\begin{array}{l}454 \\
271\end{array}$ & $\begin{array}{l}196 \\
123\end{array}$ & $\begin{array}{l}0.76 \\
0.71\end{array}$ \\
\hline J1203+0414 & B1200+045 & QSO & 1.221 & $5.96 h^{-1}$ & 72 & $429 h^{-1}$ & $590 \pm 21$ & $\sim 22$ & $\begin{array}{l}\text { A } \\
\text { B } \\
\text { C }\end{array}$ & $\begin{array}{c}850 \\
81 \\
35\end{array}$ & $\begin{array}{c}423 \\
24 \\
14\end{array}$ & $\begin{array}{l}0.63 \\
1.10 \\
0.83\end{array}$ \\
\hline $\mathrm{J} 1352+0232$ & B1349+027 & G & 0.607 & $4.83 h^{-1}$ & 188 & $908 h^{-1}$ & $458 \pm 10$ & $\sim 56$ & $\begin{array}{l}\mathrm{A} \\
\mathrm{B}\end{array}$ & $\begin{array}{l}480 \\
120\end{array}$ & $\begin{array}{c}189 \\
13\end{array}$ & $\begin{array}{l}0.84 \\
2.00\end{array}$ \\
\hline $\begin{array}{l}\mathrm{J} 1352+1107 \\
\mathrm{~J} 1600-0037\end{array}$ & $\begin{array}{c}4 \mathrm{C}+11.46 \\
\mathrm{~B} 1557-004\end{array}$ & $\begin{array}{l}\mathrm{G} \\
\mathrm{G}\end{array}$ & 0.891 & $5.61 h^{-1}$ & $\begin{array}{l}21 \\
65\end{array}$ & $118 h^{-1}$ & $\begin{array}{l}403 \pm 6 \\
190 \pm 5\end{array}$ & $\begin{array}{l}\sim 75 \\
\sim 11\end{array}$ & $\begin{array}{l}\text { all } \\
\text { A } \\
\text { B }\end{array}$ & $\begin{array}{l}598 \\
607 \\
255\end{array}$ & $\begin{array}{c}102 \\
85 \\
75\end{array}$ & $\begin{array}{l}1.59 \\
1.77 \\
1.10\end{array}$ \\
\hline $\mathrm{J} 2058+0540$ & $4 C+05.78$ & G & 1.381 & $6.04 h^{-1}$ & 153 & $923 h^{-1}$ & $351 \pm 18$ & $\sim 37$ & $\begin{array}{l}\mathrm{C} \\
\mathrm{A} \\
\mathrm{B}\end{array}$ & $\begin{array}{c}- \\
513 \\
403\end{array}$ & $\begin{array}{c}4 \\
134 \\
86\end{array}$ & $\begin{array}{c}- \\
1.21 \\
1.39\end{array}$ \\
\hline
\end{tabular}

sharply and obtain the spectral indices of components between $1.6 \mathrm{GHz}$ and $5 \mathrm{GHz}$, and finally identify CSOs from the candidates.

Quite a few GPS sources having been found to have flux variability (Torniainen et al. 2007). Since 2007, we have performed flux monitoring at $4.85 \mathrm{GHz}$ with the Urumqi $25-\mathrm{m}$ radio telescope for GPS sources from the master list for which declination $>-30^{\circ}$ (Labiano et al. 2007).

Throughout the paper, the cosmological parameters $H_{0}=$ $70.1 \mathrm{~km} \mathrm{~s}^{-1} \mathrm{Mpc}^{-1}, \Omega_{\Lambda}=0.721$ and $\Omega_{\mathrm{m}}=0.279$ (Komatsu et al. 2009) are adopted, and the spectral index $\alpha$ is defined by $S \propto v^{-\alpha}$.

\section{The EVN observations and data reduction}

The $5 \mathrm{GHz}$ VLBI observations were carried out on 12 June 2008 for the first nine targets in Table 1 (proposal code EL036A) and on 25 October 2008 for the remaining three (EL036B), using the MK5 recording system with a bandwidth of $32 \mathrm{MHz}$ and a sample rate of $256 \mathrm{Mbps}$ in dual circular polarization. The EVN antennae involved were Effelsberg, Westerbork, Jodrell (Jb1), Medicina, Noto, Onsala, Torun, Hartebeesthoek, Urumqi, and Shanghai. Hartebeesthoek was not involved in EL036B because of an antenna problem. The snapshot observations of the target sources (see Table 1) were carried out in a total of about $24 \mathrm{~h}$, DA193 and OQ208 were inset as calibrators. The data correlation was performed at the Joint Institute of VLBI in Europe (JIVE).

The Astronomical Image Processing System (AIPS) software has been used for editing, apriori calibration, fringe-fitting, self-calibration, imaging, and model-fitting of the data. The uncertainty in the flux density in the VLBI image is estimated to be about $10 \%$, and the total time per source and noise level in source image are listed in Table 2.

\section{Results and comments on individual sources}

We list basic information about 12 GPS sources and some parameters derived from the VLBI images in Table 1. Columns 1 through to 13 provide source names, optical identification (G: galaxy, QSO: quasar), redshift (de Vries et al. 2007; and the literature, those with $*$ are a photometric estimated by Tinti et al. 2005), linear scale factor $\mathrm{pc} / \mathrm{mas}$ where $H_{0}=$

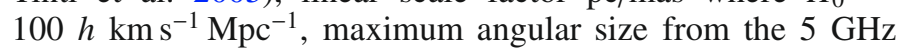
VLBI observation, maximum linear size, $4.85 \mathrm{GHz}$ total flux density measured with Urumqi single dish in December 2008 (July 2007 value for J0210+0419), the resolved-out fraction in the $5 \mathrm{GHz}$ VLBI image, the VLBI component, the integrated flux density of the component at $1.6 \mathrm{GHz}$ (from Paper I), the integrated flux density of the component at $5 \mathrm{GHz}$ (using the same restoring beam as that used in the $1.6 \mathrm{GHz}$ image), and the spectral index of component calculated from Cols. (11) and (12). More image parameters are listed in Table 2.

In the following, we summarize the VLBI results and comment on the individual sources.

\section{1. $J 0210+0419$}

The $5 \mathrm{GHz}$ VLBI image (Fig. 1) of this source shows a double lobe structure, which is similar to the $1.6 \mathrm{GHz}$ image in Paper I. The north-east lobe is more compact at $5 \mathrm{GHz}$, and the south-west one is resolved into "head-tail" components "B1" and "B2". The spectral indices of component "A" and "B" between $1.6 \mathrm{GHz}$ and $5 \mathrm{GHz}$ are both steeper $(0.86$ and 0.66 , respectively, see Table 1), indicating that they are lobes/hotspots. 
L. Cui et al.: EVN observations of twelve GPS radio sources at $5 \mathrm{GHz}$

Table 2. The component parameters of the VLBI images at $5 \mathrm{GHz}$.

\begin{tabular}{|c|c|c|c|c|c|c|c|c|c|c|c|}
\hline $\begin{array}{c}1 \\
\text { Name } \\
\text { \& Class }\end{array}$ & $\begin{array}{c}2 \\
T \\
(\min ) \\
\end{array}$ & $\begin{array}{c}3 \\
\mathrm{rms} \\
(\mathrm{mJy} / \mathrm{b})\end{array}$ & $\begin{array}{c}4 \\
S_{\text {vlbi }} \\
(\mathrm{mJy}) \\
\end{array}$ & $\begin{array}{c}5 \\
\text { Comp. }\end{array}$ & $\begin{array}{c}6 \\
S_{\mathrm{p}} \\
(\mathrm{mJy} / \mathrm{b})\end{array}$ & $\begin{array}{c}7 \\
S_{\text {int }} \\
(\mathrm{mJy}) \\
\end{array}$ & $\begin{array}{c}8 \\
\theta_{1} \times \theta_{2} \\
(\mathrm{mas} \times \mathrm{mas}) \\
\end{array}$ & $\begin{array}{c}9 \\
\mathrm{PA} \\
\left({ }^{\circ}\right) \\
\end{array}$ & $\begin{array}{c}10 \\
D \\
\text { (mas) } \\
\end{array}$ & $\begin{array}{l}11 \\
\text { PA } \\
\left({ }^{\circ}\right) \\
\end{array}$ & $\begin{array}{c}12 \\
T_{\mathrm{b}} \\
\left(\times 10^{8} \mathrm{~K}\right) \\
\end{array}$ \\
\hline J0210+0419 & 50 & 0.8 & 243 & A & 94 & 141 & $3.2 \times 2.0$ & 1.4 & 0 & & 11.8 \\
\hline \multirow[t]{2}{*}{$\mathrm{CSO}$} & & & & B1 & 28 & 65 & $4.2 \times 3.8$ & 176 & $71.4 \pm 0.1$ & $-152.7 \pm 0.1$ & 2.2 \\
\hline & & & & B2 & 22 & 63 & $6.3 \times 2.9$ & 38 & $67.1 \pm 0.1$ & $-152.7 \pm 0.1$ & 1.8 \\
\hline J0323+0534 & 149 & 0.2 & 135 & A1 & 19 & 19 & $5.0 \times 1.0$ & 83 & 0 & & 0.3 \\
\hline \multirow[t]{2}{*}{$\mathrm{CSOc}$} & & & & A2 & 42 & 109 & $16.8 \times 5.1$ & 59 & $5.2 \pm 0.1$ & $42.9 \pm 0.1$ & 0.3 \\
\hline & & & & A3 & 8 & 16 & $12.9 \times 3.8$ & 63 & $22.8 \pm 0.1$ & $57.4 \pm 0.1$ & 0.1 \\
\hline J0433-0229 & 162 & 0.8 & 449 & A1 & 270 & 388 & $5.0 \times 3.8$ & 161 & 0 & & 6.7 \\
\hline \multirow[t]{2}{*}{$\mathrm{CSOc} / \mathrm{cj}$} & & & & A2 & 30 & 35 & $3.7 \times 1.3$ & 157 & $13.0 \pm 0.1$ & $166.4 \pm 0.1$ & 2.4 \\
\hline & & & & B & 9 & 15 & $7.9 \times 3.2$ & 156 & $52.6 \pm 0.1$ & $154.8 \pm 0.1$ & 0.2 \\
\hline J0913+1454 & 108 & 0.2 & 253 & A1 & 24 & 59 & $2.9 \times 1.5$ & 105 & 0 & & 4.0 \\
\hline \multirow[t]{8}{*}{$\mathrm{CSOc}$} & & & & A2 & 11 & 37 & $3.7 \times 2.6$ & 116 & $1.4 \pm 0.1$ & $-81.8 \pm 0.4$ & 1.0 \\
\hline & & & & A3 & 3 & 5 & $3.5 \times 1.6$ & 169 & $5.5 \pm 0.1$ & $-77.0 \pm 0.4$ & 0.3 \\
\hline & & & & A4 & 17 & 41 & $2.7 \times 2.3$ & 72 & $10.6 \pm 0.1$ & $-85.5 \pm 0.1$ & 2.0 \\
\hline & & & & A5 & 16 & 41 & $3.0 \times 2.0$ & 94 & $11.5 \pm 0.1$ & $-86.9 \pm 0.1$ & 2.0 \\
\hline & & & & A6 & 11 & 21 & $2.2 \times 1.6$ & 69 & $5.6 \pm 0.1$ & $91.6 \pm 0.1$ & 2.0 \\
\hline & & & & A7 & 3 & 9 & $4.2 \times 3.0$ & 25 & $6.6 \pm 0.1$ & $91.7 \pm 0.4$ & 0.2 \\
\hline & & & & A8 & 2 & 3 & $2.8 \times 1.1$ & 37 & $11.8 \pm 0.1$ & $88.8 \pm 0.3$ & 0.3 \\
\hline & & & & B & 19 & 47 & $3.0 \times 0.6$ & 117 & $54.1 \pm 0.1$ & $71.7 \pm 0.1$ & 8.0 \\
\hline $\begin{array}{c}\mathrm{J} 1057+0012 \\
\mathrm{cj}\end{array}$ & 84 & 0.3 & 316 & A & 183 & 289 & $3.1 \times 1.4$ & 36 & 0 & & 23.0 \\
\hline $\mathrm{J} 1109+1043$ & 90 & 0.2 & 386 & A & 173 & 278 & $4.4 \times 1.7$ & 58 & 0 & & 12.0 \\
\hline $\mathrm{CSOc}$ & & & & B & 55 & 88 & $4.3 \times 1.7$ & 65 & $46.3 \pm 0.1$ & $103.3 \pm 0.1$ & 4.0 \\
\hline J1135-0021 & 60 & 0.7 & 358 & A1 & 134 & 185 & $3.3 \times 2.2$ & 171 & 0 & & 11.0 \\
\hline \multirow[t]{5}{*}{ CSO } & & & & A2 & 29 & 69 & $7.4 \times 2.4$ & 135 & $3.3 \pm 0.5$ & $165.0 \pm 4.2$ & 1.6 \\
\hline & & & & A3 & 9 & 27 & $10.5 \times 2.2$ & 41 & $14.5 \pm 1.9$ & $165.5 \pm 3.5$ & 0.5 \\
\hline & & & & B1 & 44 & 79 & $6.0 \times 2.4$ & 176 & $94.6 \pm 0.3$ & $163.3 \pm 0.1$ & 2.3 \\
\hline & & & & B2 & 35 & 91 & $8.8 \times 3.0$ & 158 & $91.4 \pm 0.5$ & $162.7 \pm 0.1$ & 1.5 \\
\hline & & & & B3 & 19 & 69 & $13.6 \times 2.6$ & 154 & $86.6 \pm 1.2$ & $163.6 \pm 0.2$ & 0.8 \\
\hline $\mathrm{J} 1203+0414$ & 48 & 0.2 & 461 & A1 & 260 & 396 & $2.5 \times 1.1$ & 118 & 0 & & 68.4 \\
\hline \multirow[t]{3}{*}{$c j$} & & & & A 2 & 25 & 56 & $4.5 \times 1.3$ & 126 & $4.3 \pm 0.1$ & $119.5 \pm 0.1$ & 2.0 \\
\hline & & & & B & 11 & 15 & $1.9 \times 1.1$ & 94 & $14.6 \pm 0.1$ & $101.4 \pm 0.1$ & 3.0 \\
\hline & & & & $\mathrm{C}$ & 8 & 12 & $2.8 \times 0.4$ & 111 & $63.3 \pm 0.1$ & $104.4 \pm 0.1$ & 5.0 \\
\hline $\mathrm{J} 1352+0232$ & 45 & 0.2 & 202 & A1 & 90 & 106 & $3.6 \times 1.7$ & 95 & 0 & & 6.0 \\
\hline \multirow[t]{2}{*}{$\mathrm{CSOc}$} & & & & $\mathrm{A} 2$ & 57 & 68 & $4.3 \times 1.7$ & 59 & $7.1 \pm 0.1$ & $-133.6 \pm 0.1$ & 3.0 \\
\hline & & & & B & 10 & 13 & $4.9 \times 1.2$ & 168 & $170.1 \pm 0.1$ & $-112.6 \pm 0.1$ & 0.8 \\
\hline J1352+1107 & 54 & 0.5 & 102 & A & 29 & 77 & $9.2 \times 3.7$ & 138 & 0 & & 1.0 \\
\hline \multirow[t]{2}{*}{$\mathrm{cx}$} & & & & B & 13 & 60 & $14.3 \times 5.4$ & 39 & $5.2 \pm 0.1$ & $56.4 \pm 0.1$ & 0.3 \\
\hline & & & & $\mathrm{C}$ & 15 & 46 & $12.4 \times 2.8$ & 20 & $4.8 \pm 0.1$ & $-39.7 \pm 0.1$ & 0.5 \\
\hline J1600-0037 & 103 & 0.2 & 169 & A & 70 & 83 & $2.5 \times 2.2$ & 130 & 0 & & \\
\hline \multirow[t]{3}{*}{$\mathrm{cj}$} & & & & B1 & 29 & 57 & $10.0 \times 1.0$ & 69 & $31.8 \pm 0.1$ & $84.6 \pm 0.1$ & \\
\hline & & & & B2 & 21 & 46 & $10.9 \times 1.2$ & 80 & $25.2 \pm 0.1$ & $87.5 \pm 0.1$ & \\
\hline & & & & $\mathrm{C}$ & 4 & 4 & $1.8 \times 1.1$ & 72 & $50.8 \pm 0.1$ & $77.8 \pm 0.1$ & \\
\hline $\mathrm{J} 2058+0540$ & 47 & 0.4 & 220 & A1 & 104 & 124 & $4.5 \times 1.0$ & 9.3 & 0 & & 14.0 \\
\hline \multirow[t]{2}{*}{$\mathrm{CSO}$} & & & & A2 & 8.2 & 13 & $7.7 \times 2.1$ & 177 & $15.5 \pm 0.1$ & $-175.0 \pm 0.2$ & 0.4 \\
\hline & & & & B & 42 & 68 & $8.3 \times 2.4$ & 172 & $132.6 \pm 0.1$ & $171.9 \pm 0.1$ & 2.0 \\
\hline
\end{tabular}

Notes. The columns provide: (1) source name and classification (CSOc: CSO candidate, cj: core-jet, cx: complex); (2) total observing time per source in minutes; (3) noise level per beam in $5 \mathrm{GHz}$ VLBI image; (4) total VLBI flux density; (5) component identification labelled to Paper I; (6), (7) peak and integral intensity of a fitted Gaussian component at $5 \mathrm{GHz}$ with the AIPS task JMFIT; (8), (9) major/minor axes and position angle of the component at $5 \mathrm{GHz}$; (10), (11) distance and position angle relative to the first component; and (12) brightness temperature of the component.

The core of the source is not detected. The total flux density is slightly lower than for PKS90 data at $5 \mathrm{GHz}$ in Table 3. According to the double lobes and tail morphologies at both 1.6 GHz and $5 \mathrm{GHz}$ and the steep spectra of the lobes, we classify the source as a CSO.

\section{2. $J 0323+0534$}

The $83 \%$ of the total flux density at $5 \mathrm{GHz}$ (Table 1 ) is resolvedout in the $5 \mathrm{GHz}$ VLBI image (Fig. 2), confirming that the source is very diffuse as suggested in Paper I. The southern diffuse component " $\mathrm{B}$ " in the $1.6 \mathrm{GHz}$ VLBI image (Paper I) is resolved-out completely in the $5 \mathrm{GHz}$ VLBI image. The north region " $\mathrm{A}$ ", which is very diffuse at $1.6 \mathrm{GHz}$, is resolved into three components "A1", "A2", and "A3", which appear to have a "core-jet" shape at $5 \mathrm{GHz}$; this leads to a very steep spectral index $\alpha_{1.65}^{5}$ of the component "A", but "A1", "A2, and "A3" are most likely types of hotspots rather than a core-jet. The total flux density is stable compared to PKS90 data at $5 \mathrm{GHz}$ in Table 3, hence we keep this source as a CSO candidate. 
Table 3. Flux density and possible variability of the 12 GPS sources.

\begin{tabular}{|c|c|c|c|c|c|c|c|c|c|}
\hline Source & $\begin{array}{l}S_{\text {PKS }} \\
(\mathrm{mJy})\end{array}$ & $\begin{array}{l}S_{\mathrm{Jan} 07} \\
(\mathrm{mJy})\end{array}$ & $\begin{array}{l}S_{\text {Jul07 }} \\
(\mathrm{mJy})\end{array}$ & $\begin{array}{l}S_{\text {Dec08 }} \\
(\mathrm{mJy})\end{array}$ & $\begin{array}{l}S_{\text {Jul09 }} \\
(\mathrm{mJy})\end{array}$ & $\begin{array}{l}S_{\text {Nov09 }} \\
(\mathrm{mJy})\end{array}$ & $\begin{array}{c}\overline{S_{U r}} \\
(\mathrm{mJy})\end{array}$ & $\begin{array}{c}\sigma_{S_{U r}} / \overline{S_{U r}} \\
(\%)\end{array}$ & $\begin{array}{c}\left.\overline{S_{U r}}-S_{\mathrm{PKS}}\right) / S_{\mathrm{PKS}} \\
(\%)\end{array}$ \\
\hline J0210+0419 & 300 & $302 \pm 10$ & $282 \pm 2$ & & $274 \pm 4$ & $298 \pm 4$ & 289 & 4.0 & -3.7 \\
\hline $\mathrm{J} 0323+0534$ & 830 & $868 \pm 9$ & $833 \pm 5$ & $796 \pm 21$ & $814 \pm 10$ & $836 \pm 4$ & 829 & 2.9 & -0.1 \\
\hline J0433-0229 & 640 & $637 \pm 14$ & $705 \pm 25$ & $612 \pm 5$ & & & 651 & 6.0 & 1.8 \\
\hline J0913+1454 & 300 & $297 \pm 8$ & $289 \pm 3$ & $275 \pm 18$ & & $311 \pm 6$ & 293 & 4.5 & -2.3 \\
\hline $\mathrm{J} 1057+0012$ & 370 & $351 \pm 6$ & $369 \pm 4$ & $355 \pm 21$ & $345 \pm 4$ & $373 \pm 14$ & 359 & 3.0 & -3.1 \\
\hline $\mathrm{J} 1109+1043$ & 400 & $428 \pm 8$ & $416 \pm 5$ & $420 \pm 7$ & $434 \pm 4$ & $414 \pm 7$ & 422 & 1.8 & 5.6 \\
\hline J1135-0021 & 440 & $427 \pm 8$ & $392 \pm 4$ & $422 \pm 13$ & & & 414 & 3.7 & -6.0 \\
\hline $\mathrm{J} 1203+0414$ & 520 & $611 \pm 7$ & $561 \pm 3$ & $590 \pm 21$ & $604 \pm 4$ & $632 \pm 5$ & 600 & 3.9 & 15.3 \\
\hline $\mathrm{J} 1352+0232$ & 470 & $469 \pm 7$ & $453 \pm 4$ & $458 \pm 10$ & $470 \pm 4$ & $465 \pm 6$ & 463 & 1.4 & -1.5 \\
\hline $\mathrm{J} 1352+1107$ & 410 & $418 \pm 5$ & $406 \pm 3$ & $403 \pm 6$ & $411 \pm 3$ & $432 \pm 3$ & 414 & 2.5 & 1.0 \\
\hline J1600-0037 & 180 & $212 \pm 3$ & $177 \pm 5$ & $190 \pm 5$ & & & 193 & 7.5 & 7.2 \\
\hline $\mathrm{J} 2058+0540$ & 340 & & $340 \pm 3$ & $351 \pm 18$ & $343 \pm 4$ & $345 \pm 5$ & 345 & 1.2 & 1.4 \\
\hline
\end{tabular}

Notes. Column 2 is the PKS90 flux density at $5.0 \mathrm{GHz}$. Columns 3 to 7 indicate the $4.85 \mathrm{GHz}$ flux density measured with the Urumqi telescope in January 2007, July 2007, December 2008, July 2009, and November 2009, respectively; Cols. 8-10 give the mean flux density of the Urumqi data, the standard deviation of the Urumqi data divided by the mean value, and the relative variation between the mean flux density and the PKS90 data.

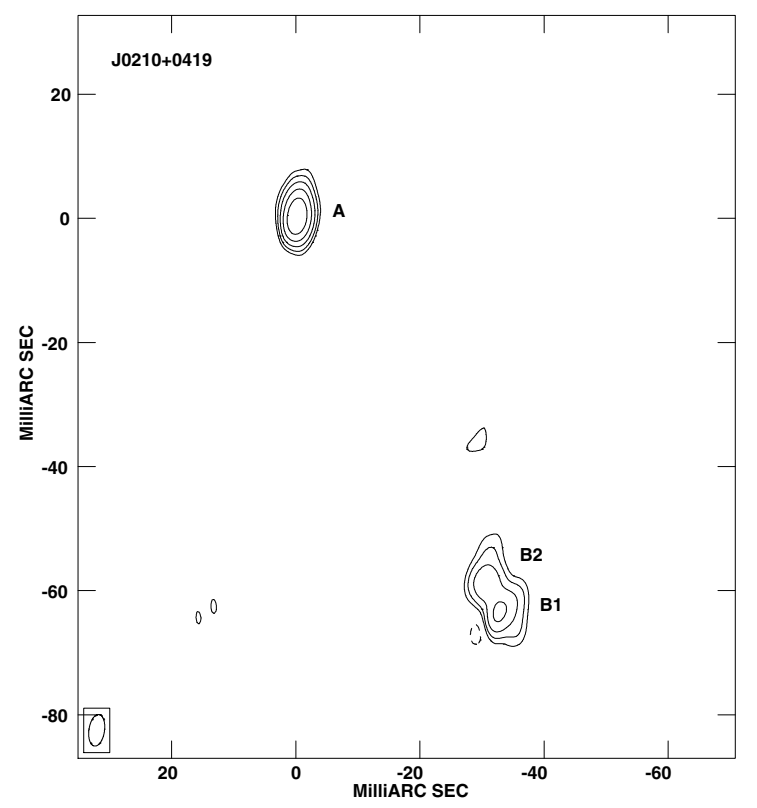

Fig. 1. J0210+0419: the restoring beam is $5.1 \times 2.5$ mas in PA $-8.2^{\circ}$, the contours are $3 \mathrm{mJy} /$ beam times $-1,1,2,4,8$, and 16 , and the peak flux density is $95 \mathrm{mJy} / \mathrm{beam}$.

\section{3. $J 0433-0229$}

The major component "A" of $1.6 \mathrm{GHz}$ image (Paper I) is resolved into "A1" and "A2" at $5 \mathrm{GHz}$ (Fig. 3), and the weak component " $\mathrm{B}$ " is marginally detected. The overall shape of the source at $5 \mathrm{GHz}$ is similar to that at $1.6 \mathrm{GHz}$, resembling a core-jet. Both of the components "A" and "B" however, have steep spectra (Table 1), and about $27 \%$ of the total flux density is resolvedout in the $5 \mathrm{GHz}$ image. The total flux density is stable relative to PKS90 data at $5 \mathrm{GHz}$ in Table 3 . This source may be a corejet one or a CSO candidate, although additional high sensitivity VLBI observation would be needed to clarify this.

\section{4. $J 0913+1454$}

The $5 \mathrm{GHz}$ VLBI image (Fig. 4) of this source can be resolved into several components "A1" to "A8" in the region " $\mathrm{A}$ ", and

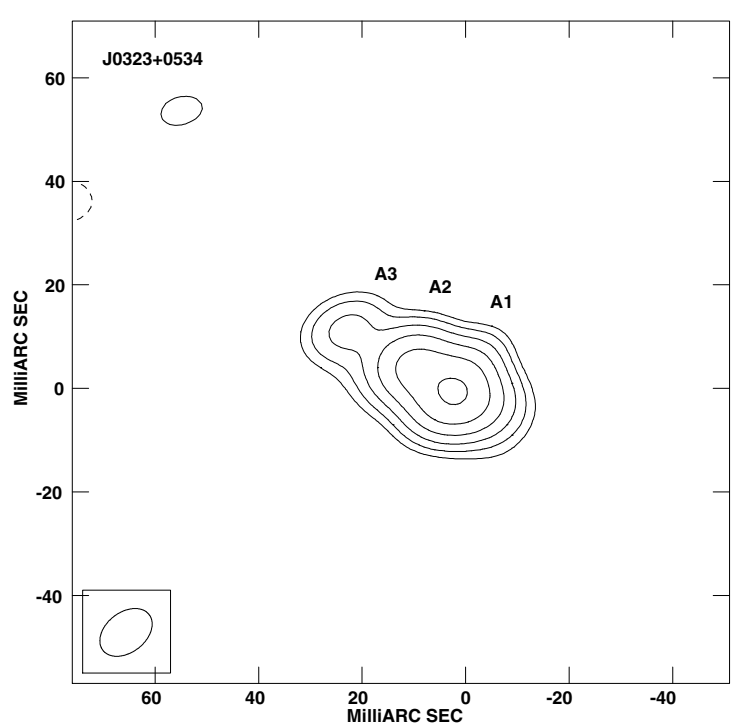

Fig. 2. J0323+0534: the restoring beam is $11.3 \times 7.7$ mas in PA $-52.3^{\circ}$, the contours are $1.5 \mathrm{mJy} /$ beam times $-1,1,2,4,8,16$, and 32 , and the peak flux density is $54 \mathrm{mJy} / \mathrm{beam}$.

the component "B", which is more compact at $5 \mathrm{GHz}$ than at $1.6 \mathrm{GHz}$ (Paper I). It is difficult to classify the source, the components "A1" to "A8" most likely representing a core and a set of jets. The core may be at either "A8" or "A5": in the former case the source may be a CSO, in the latter the source may be a core-jet. The spectral index $\alpha_{1.65}^{5}$ of component "A" (integrated) and " $\mathrm{B}$ " is 0.79 and 1.13 , respectively. The total flux density is stable compared to PKS90 data at $5 \mathrm{GHz}$ in Table 3.

\section{5. $J 1057+0012$}

The $5 \mathrm{GHz}$ image (Fig. 5) contains a point-like source, the possible jet in the $1.6 \mathrm{GHz}$ image (Paper I) being neither detected nor resolved-out. The spectral index $\alpha_{1.65}^{5}$ of main component is 0.55 , which is flatter than that of a typical lobe component in other sources. The total flux density is slightly lower than that of the PKS90 data at $5 \mathrm{GHz}$ in Table 3 . We classify it as a core-jet source on the basis of both the $1.6 \mathrm{GHz}$ and $5 \mathrm{GHz}$ images. 
L. Cui et al.: EVN observations of twelve GPS radio sources at $5 \mathrm{GHz}$

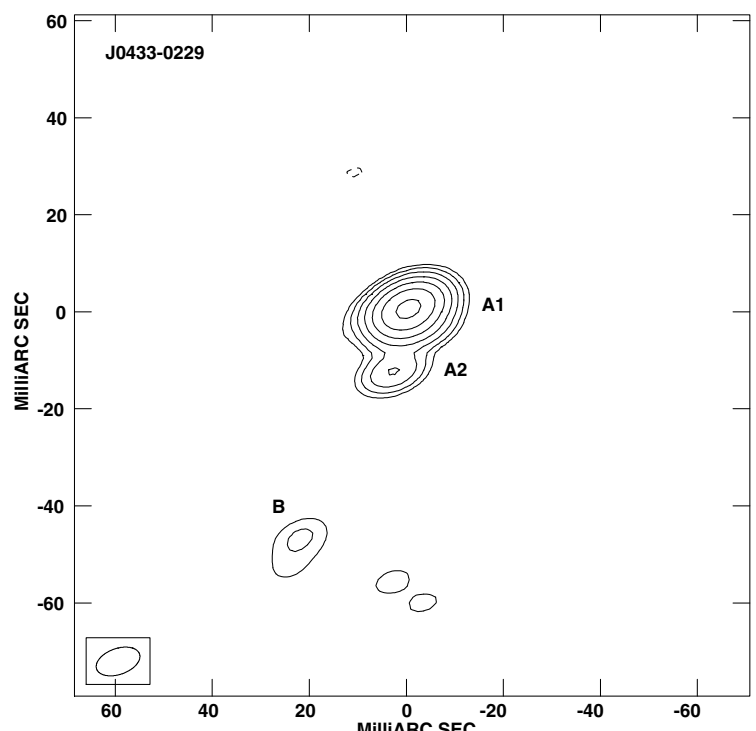

Fig. 3. J0433-0229: the restoring beam is $9.4 \times 5.3$ mas in PA $-70.5^{\circ}$, the contours are $3.5 \mathrm{mJy} /$ beam times $-2,1,2,4,8,16,32$, and 64 , and the peak flux density is $270 \mathrm{mJy} /$ beam.

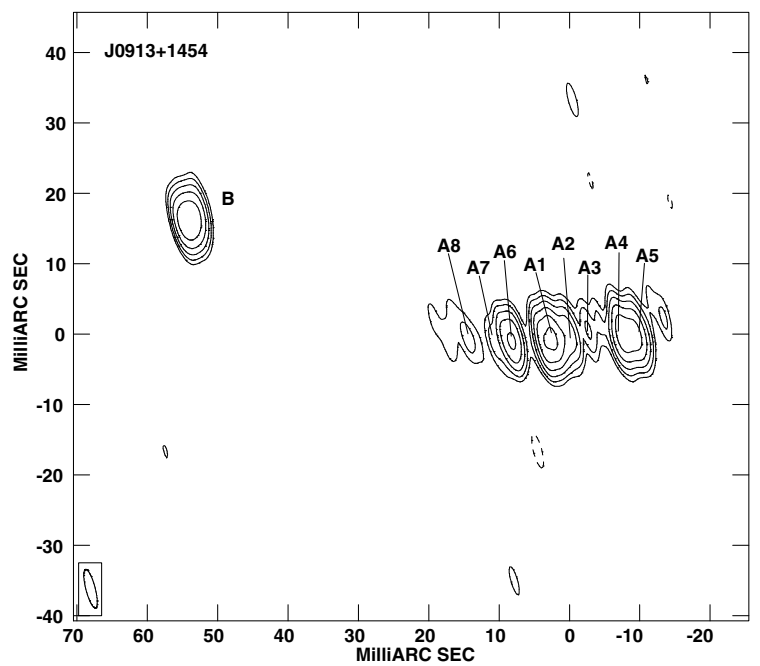

Fig. 4. J0913+1454: the restoring beam is $5.7 \times 1.3$ mas in PA $14.5^{\circ}$, the contours are $0.6 \mathrm{mJy} /$ beam times $-2,1,2,4,8,16$, and 32 , and the peak flux density is $24 \mathrm{mJy} / \mathrm{beam}$.

\section{6. $J 1109+1043$}

The 5 GHz VLBI image (Fig. 6) of this source exhibits a double structure, which is similar to the $1.6 \mathrm{GHz}$ image (Paper I). Both components have equally steep spectra between $1.6 \mathrm{GHz}$ and $5 \mathrm{GHz}$, of index 1.13 for component " $\mathrm{A}$ " and 1.11 for component "B", whose values indicate that they are lobs/hotspots. The total flux density is slightly higher than that of the PKS90 data at $5 \mathrm{GHz}$ in Table 3 . The source is most likely a CSO or a compact double (CD) since no tail/jet emission has been detected.

\subsection{J1135-0021}

The $5 \mathrm{GHz}$ VLBI image (Fig. 7) shows a double-lobe structure, which is similar to that in $1.6 \mathrm{GHz}$ image (Paper I). The lobes/tails can be fitted by several subcomponents ("A1", "A2", "A3" for "A", and "B1", "B2", "B3" for "B"). The spectral indices of " $A$ " and "B" between $1.6 \mathrm{GHz}$ and $5 \mathrm{GHz}$ are equally

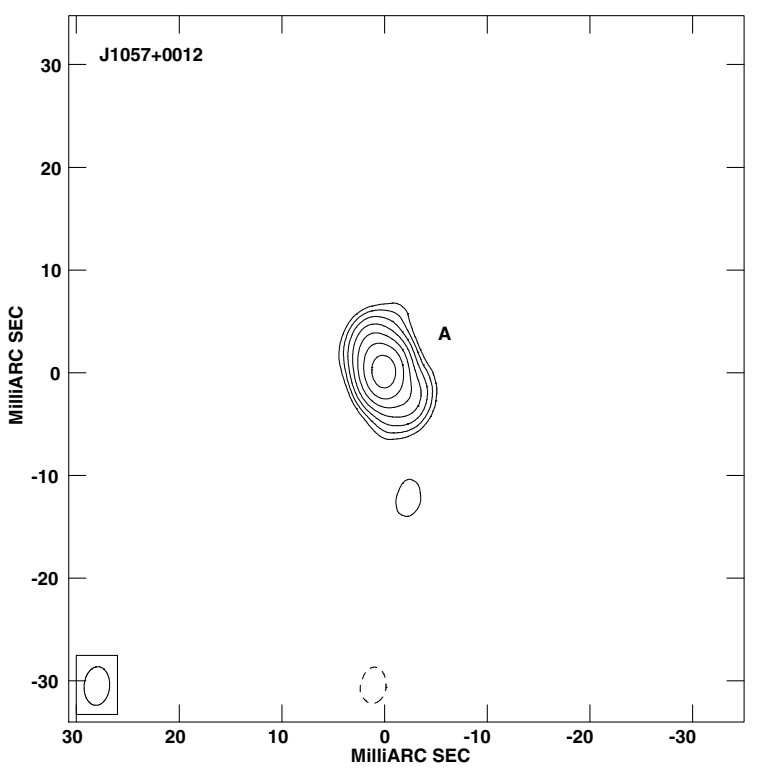

Fig. 5. J1057+0012: the restoring beam is $3.8 \times 2.5$ mas in PA $-4.7^{\circ}$, the contours are $2 \mathrm{mJy} /$ beam times $-1,1,2,4,8,16,32$, and 64 , and the peak flux density is $192 \mathrm{mJy} /$ beam.

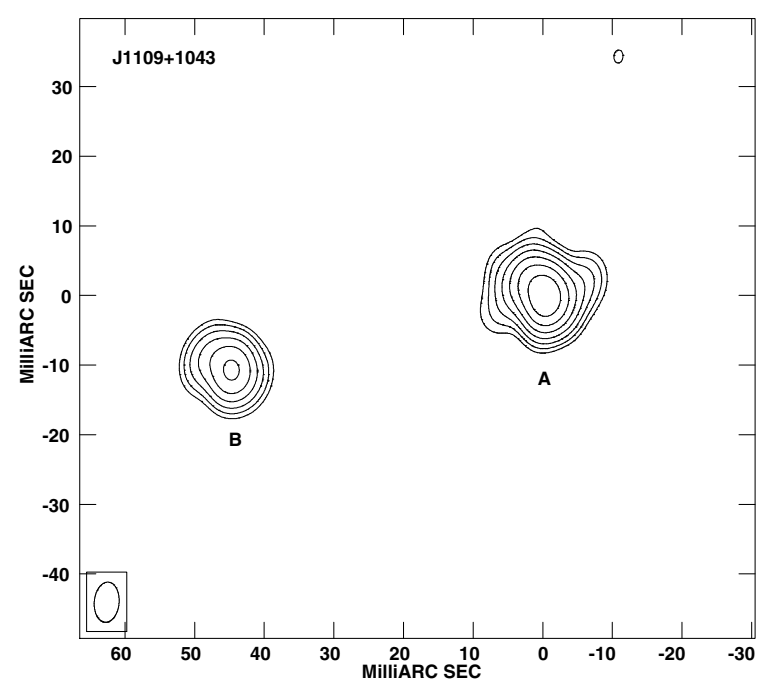

Fig. 6. J1109+1043: the restoring beam is $5.8 \times 3.6$ mas in PA $-5^{\circ}$, the contours are $1.5 \mathrm{mJy} /$ beam times $-1,1,2,4,8,16,32$, and 64 , and the peak flux density is $178 \mathrm{mJy} /$ beam.

steep, and the linear size is smaller than $1 \mathrm{kpc} h^{-1}$ (Table 1). The total flux density is slightly lower than for the PKS90 data at $5 \mathrm{GHz}$ in Table 3. We confirm that the source is a CSO.

\section{8. $J 1203+0414$}

This is a GPS quasar, and the $5 \mathrm{GHz}$ VLBI image (Fig. 8) exhibits a core-jet like structure. The component "A1" probably presents the core of the source, since the spectral index 0.63 of "A" corresponds to a flatter spectrum than those of other components " $\mathrm{B}$ " and "C". The total flux density is higher by $15.3 \%$ than the PKS90 data at $5 \mathrm{GHz}$ in Table 3, and it is resolved-out by about $22 \%$ in the $5 \mathrm{GHz}$ VLBI image, some weak jets possibly being resolved. We classify the quasar as a core-jet source. 


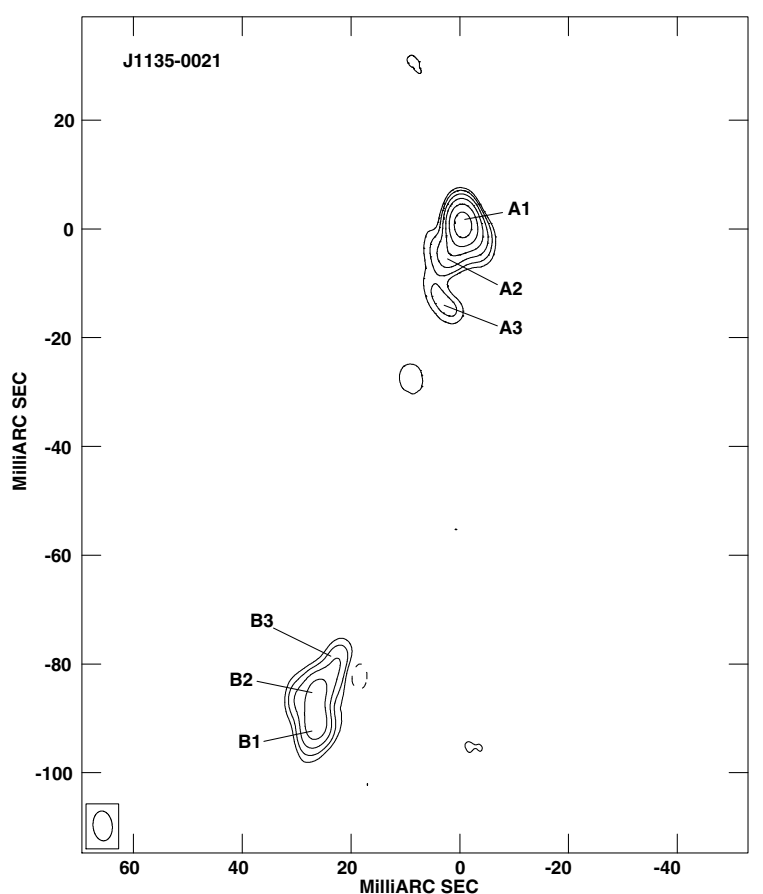

Fig. 7. J1135-0021: the restoring beam is $5.5 \times 3.5$ mas in PA $5.7^{\circ}$, the contours are $2.8 \mathrm{mJy} /$ beam times $-1,1,2,4,8,16$, and 32 , and the peak flux density is $137 \mathrm{mJy} / \mathrm{beam}$.

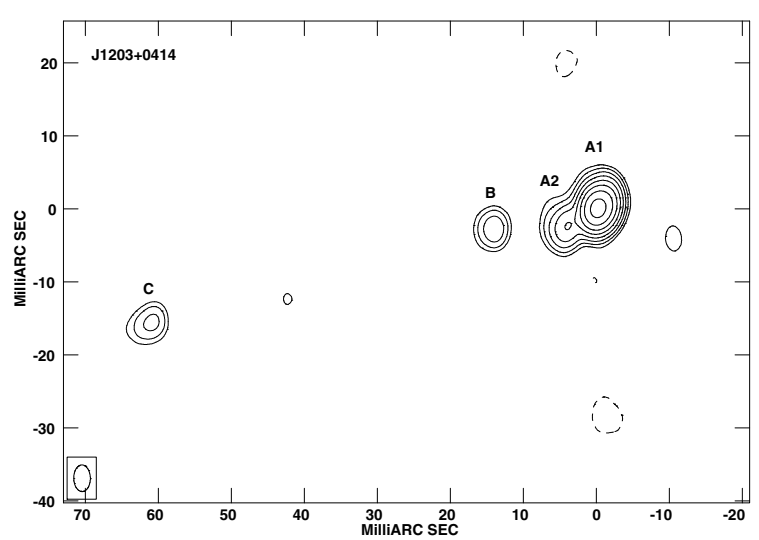

Fig. 8. J1203+0414: the restoring beam is $3.7 \times 2.3$ mas in PA $0.9^{\circ}$, the contours are $1.5 \mathrm{mJy} /$ beam times $-1,1,2,4,8,16,32,64$, and 128 , and the peak flux density is $262 \mathrm{mJy} / \mathrm{beam}$.

\section{9. $J 1352+0232$}

As in the $1.6 \mathrm{GHz}$ VLBI image in Paper I, the $5 \mathrm{GHz}$ VLBI image (Fig. 9) exhibits a strong component "A" and a weak component "B". The bright component "A" can be fitted with two close components "A1" and "A2". The components " $A$ " and "B" have the integrated spectral indices $\alpha_{1.65}^{5}$ of 0.84 and 2.0, respectively. The total flux density of the source is resolved-out by about $56 \%$ in the $5 \mathrm{GHz}$ VLBI image. The total flux density is stable compared with PKS90 data at $5 \mathrm{GHz}$ in Table 3. The source could be a CSO if it were really a double-lobe source, or a core-jet source if the core resided in the component "A1".

\subsection{J1352+1107}

The $5 \mathrm{GHz}$ VLBI image of the source detected a complex feature (Fig. 10), which is fitted with three components "A", "B", and "C". About $75 \%$ of the total flux density (Table 1) is resolved-out

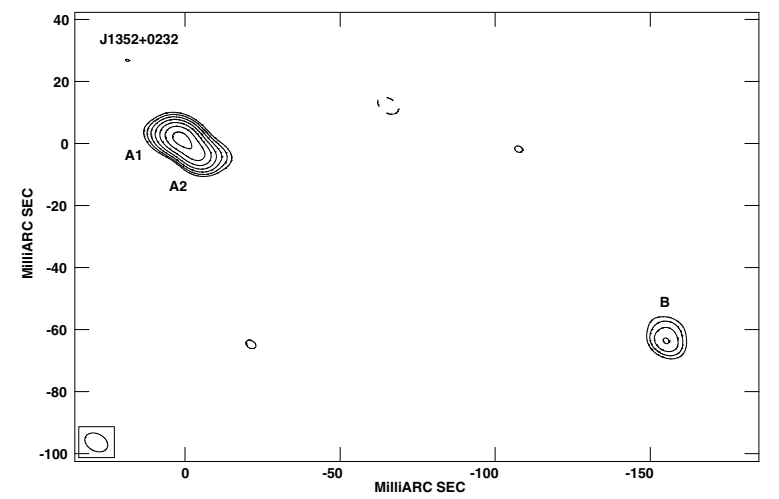

Fig. 9. J1352+0232: the restoring beam is $7.8 \times 5.5$ mas in PA $62.3^{\circ}$, the contours are $1.2 \mathrm{mJy} /$ beam times $-1,1,2,4,8,16,32$, and 64 , and the peak flux density is $102 \mathrm{mJy} / \mathrm{beam}$.

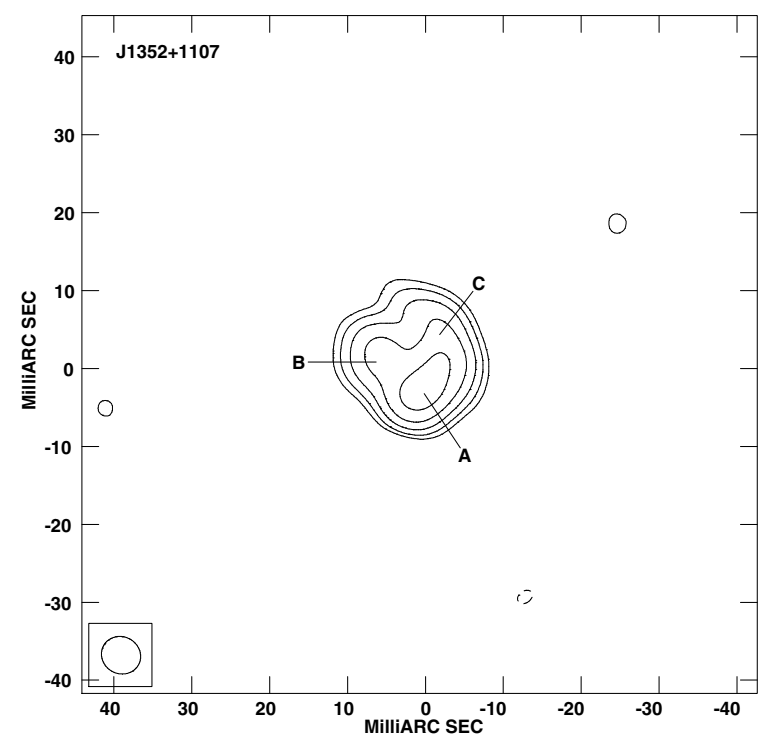

Fig. 10. J1352+1107: the restoring beam is $5.1 \times 4.7$ mas in PA $61.1^{\circ}$, the contours are $1.2 \mathrm{mJy} /$ beam times $-1,1,2,4,8$, and 16 , and the peak flux density is $29 \mathrm{mJy} / \mathrm{beam}$.

in the $5 \mathrm{GHz}$ VLBI image, confirming that the source is very diffuse as suggested in paper I. The whole source exhibits a steep spectrum of 1.59 between $1.6 \mathrm{GHz}$ and $5 \mathrm{GHz}$. The total flux density is stable compared to PKS90 data at $5 \mathrm{GHz}$ in Table 3. With the current data in hand, it is difficult to classify this source; both a core and curving jets are probably embedded in the diffuse emission.

\subsection{J1600-0037}

The $5 \mathrm{GHz}$ VLBI image (Fig. 11) has a core-jet like feature, which consists of a bright component "A", components "B1"/"B2", and a weak component "C". The spectral index of the component " $\mathrm{A}$ " between $1.6 \mathrm{GHz}$ and $5 \mathrm{GHz}$ is 1.77 . It is difficult to identify the core because the " $\mathrm{B}$ " is not clearly resolved in the $1.6 \mathrm{GHz}$ VLBI image (Paper I), the component "B1" in the $5 \mathrm{GHz}$ VLBI image probably being a candidate core. The weak component " $\mathrm{C}$ " emerging in the $5 \mathrm{GHz}$ image, which is not detected in $1.6 \mathrm{GHz}$ image (probably because of low-frequency absorption), is possibly a counter-jet. The total flux density has a variability of $7.5 \%$ in $2007-2009$ and a variation of $7.2 \%$ relative to PKS90 data at $5 \mathrm{GHz}$ in Table 3 . We therefore classify the 


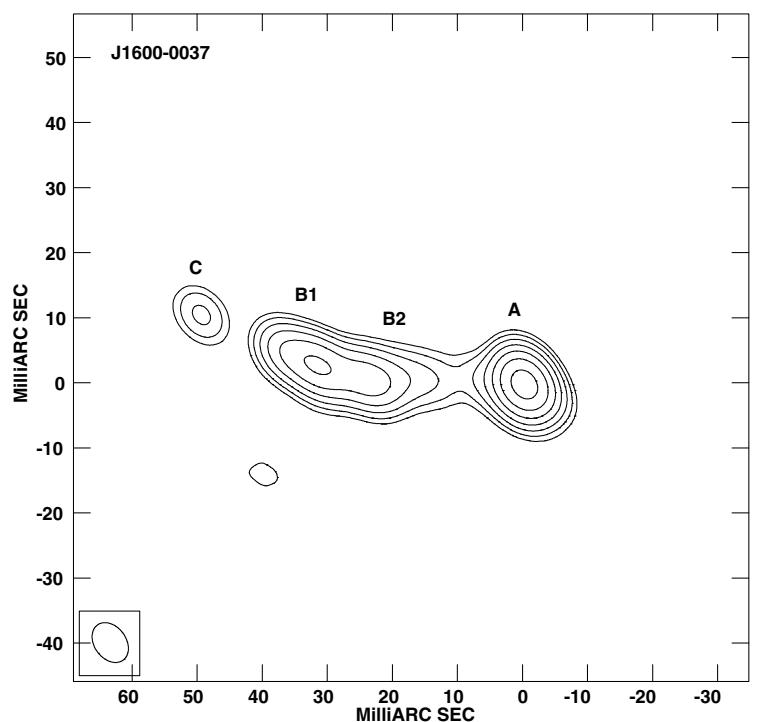

Fig. 11. J1600-0037: the restoring beam is $6.7 \times 4.9$ mas in PA $36.3^{\circ}$, the contours are $0.8 \mathrm{mJy} /$ beam times $-1,1,2,4,8,16,32$, and 64 , and the peak flux density is $71 \mathrm{mJy} /$ beam.

source as a core-jet source on the basis of its VLBI morphology and flux variability.

\subsection{2. $J 2058+0540$}

The $5 \mathrm{GHz}$ VLBI image (Fig. 12) shows a double-lobe source, which is similar to the $1.6 \mathrm{GHz}$ image in Paper I. The northern lobe is resolved into "head-tail" components "A1" and "A2" in the $5 \mathrm{GHz}$ image. The spectral indices of components " $\mathrm{A}$ " and "B" between $1.6 \mathrm{GHz}$ and $5 \mathrm{GHz}$ are 1.21 and 1.39 , respectively. The total flux density is stable relative to the PKS90 data at $5 \mathrm{GHz}$ in Table 3 . We classify the source as a CSO. We found that $37 \%$ of the total flux density is resolved-out in the $5 \mathrm{GHz}$ image, indicating that in the source some form of jet and/or diffuse emission is missing in the VLBI image.

\section{Flux density observations of the sources at $4.85 \mathrm{GHz}$}

At high radio frequencies in particular, it has been reported that, many GPS/HFP sources exhibit significant flux variability (Torniainen et al. 2007; Orienti et al. 2007). Jauncey et al. (2003) found that $\sim 10 \%$ of GPS sources experienced flux density variability over a period of 30 months. We measured the flux densities of the GPS sources in the Labiano et al. (2007) sample at $4.85 \mathrm{GHz}$, by observing with the Urumqi $25-\mathrm{m}$ telescope between 2007 and 2009. Compared to 87GB data, Liu et al. (2009) found that $44 \%$ GPS quasars exhibit $>10 \%$ flux variation over a period of about 20 years, while this fraction is only $11 \%$ for GPS galaxies. Detailed analysis of our monitoring data will be presented in another paper, and here we just list the measured flux densities of our VLBI targets in Table 3 for epochs during which most of the VLBI targets are observed.

The flux densities were determined using antenna slews with "cross-scans" in azimuth and elevation, fourfold in each coordinate. This enabled us to check the pointing offsets in both coordinates. After applying a correction for small pointing offsets, the amplitudes of both AZ and EL were averaged. We then corrected the measurements for the elevation-dependent antenna gain and the remaining systematic time-dependent effects, using a

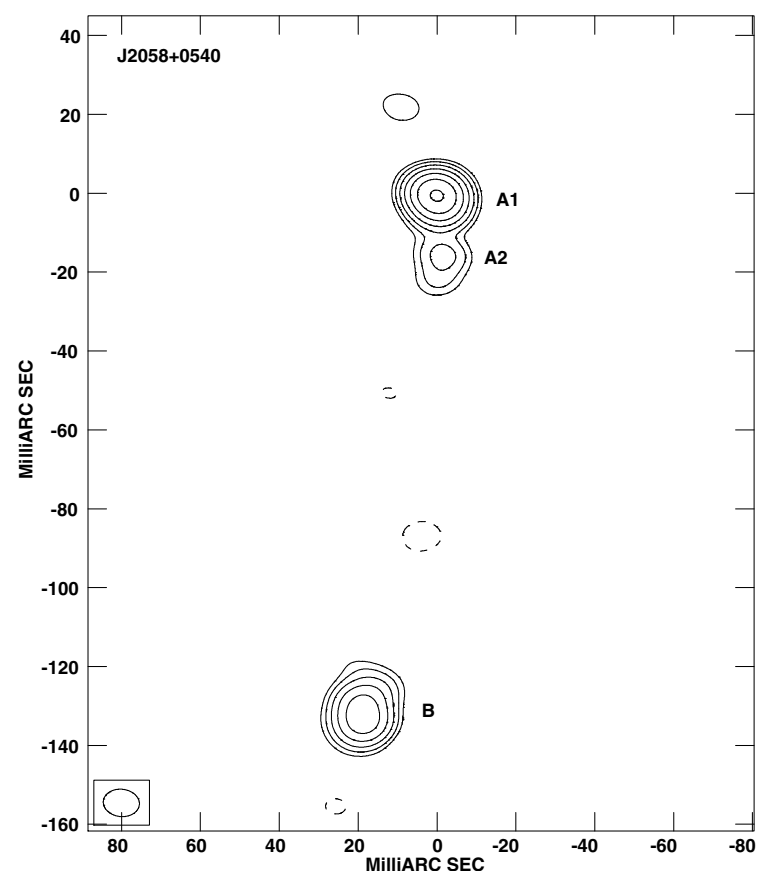

Fig. 12. J2058+0540: the restoring beam is $9.2 \times 6.9$ mas in PA $84.2^{\circ}$, the contours are $1.5 \mathrm{mJy} /$ beam times $-1,1,2,4,8,16,32$, and 64 , and the peak flux density is $105 \mathrm{mJy} /$ beam.

Table 4. The jet viewing angles estimated for the CSOs.

\begin{tabular}{lccccc}
\hline \hline Source & Type & $\Theta_{1.6 \mathrm{G}}^{k=2}$ & $\Theta_{1.6 \mathrm{G}}^{k=3}$ & $\Theta_{5 \mathrm{G}}^{k=2}$ & $\Theta_{5 \mathrm{G}}^{k=3}$ \\
\hline $\mathrm{J} 0210+0419$ & CSO & - & 37 & 48 & 60 \\
$\mathrm{~J} 1135-0021$ & CSO & 22 & 47 & 33 & 52 \\
$\mathrm{~J} 2058+0540$ & CSO & 66 & 72 & 41 & 56 \\
\hline
\end{tabular}

number of steep spectrum and non-variable secondary calibrators. Finally, we related our observations to the absolute flux density by using the scale $7.5 \mathrm{Jy}$ at $5 \mathrm{GHz}$ of the primary calibrator 3C 286 (Ott et al. 1994).

Given the flux variation measured in 2007-2009, and by comparing with PKS90 $5 \mathrm{GHz}$ data in Table 3, we find that the flux densities are quite stable or slightly different for the majority of the twelve GPS sources, but that two core-jet sources J1203+0414 (quasar) and J1600-0037 exhibit considerable variability in total flux density.

\section{Discussion}

It is interesting to estimate the jet-viewing angles for the confirmed CSOs, because the CSOs are assumed to lie nearly within the plane of sky. From the VLBI morphologies and the steep spectral indices of the VLBI components between $1.6 \mathrm{GHz}$ and $5 \mathrm{GHz}$, we can firmly classify J0210+0419, J1135-0021, and $\mathrm{J} 2058+0540$ as CSOs that display symmetric double lobes/tails. We estimated the jet viewing angle from the flux ratio between the approaching and receding lobes with the formula (Taylor \& Vermeulen 1997)

$\frac{S_{\mathrm{a}}}{S_{\mathrm{r}}}=\left(\frac{1+\beta \cos \Theta}{1-\beta \cos \Theta}\right)^{k+\alpha}$,

where $S_{\mathrm{a}} / S_{\mathrm{r}}$ is the flux ratio between the approaching (stronger) lobe and receding lobe, $\Theta$ is the viewing angle (degree) of the jet axis, $\alpha$ is the source spectral index $\alpha_{h}$ in optical-thin regime 
(from Paper I), $k=2$ or $k=3$ for a continuous or discrete jet, respectively, and $\beta$ is the lobe velocity as a fraction of the speed of light. By adopting $\beta=0.1$, which is a mean lobe velocity in CSOs (Giroletti \& Polatidis 2009), we estimated the jet viewing angles for the continuous and discrete cases at $1.6 \mathrm{GHz}$ and $5 \mathrm{GHz}$, respectively, in Table 4. From a rough estimation, we could say that the three CSOs have relatively large jet-viewing angles. Although this method should be used only for the confirmed double-lobe sources, and the lobes of the CSOs are not resolved too much in the VLBI images.

Nevertheless the majority of sources in our sample have double-lobe like morphologies and total flux densities that are quite stable over 20 years. Only 3 CSOs are firmly classified according to Fanti's suggestion that a CSO should have a core inbetween double lobes or have double lobes with twin jets/tails if a core is not detected (Fanti 2009).

Furthermore, 6 sources are resolved-out in the $5 \mathrm{GHz}$ VLBI images by more than $20 \%$ of total flux density, probably due to diffuse emission in the sources. This may lead to quite steep spectral indices of VLBI components of the sources as listed in Table 1. The spectra of all 6 sources peak at $0.4 \mathrm{GHz}$ (Snellen et al. 2002), which is close to the spectral peaks of CSS sources (see e.g., Fanti et al. 1995), so some of the sources are probably not GPS but rather CSS sources if they have larger-scale diffuse emission that is resolved-out by the VLBI observations.

\section{Summary and conclusion}

We have presented the results of $5 \mathrm{GHz}$ EVN observations of 12 GPS sources from the sample of Snellen (2002) for the first time. The source structure and spectral indices between $1.6 \mathrm{GHz}$ and $5 \mathrm{GHz}$ are obtained. We have also carried out total flux density measurements with the Urumqi $25-\mathrm{m}$ telescope at $4.85 \mathrm{GHz}$ in 2007-2009, and compared the mean flux density with the PKS90 data.

1. From the source morphologies, the component spectral indices and the total flux variability, we can firmly classify three sources J0210+0419, J1135-0021, and J2058+0540 as CSOs, and J1057+0012, J1203+0414, and J1600-0037 as core-jet sources. The others J0323+0534, J0433-0229, $\mathrm{J} 0913+1454, \mathrm{~J} 1109+1043$, and $\mathrm{J} 1352+0232$ remain as CSO candidates, and J1352+1107 is a complex VLBI feature whose nature need to clarify in the future.

2. The total flux densities of the GPS sources at $5 \mathrm{GHz}$ were quite stable in 2007-2009 and over the long term compared with the PKS90 data, except for core-jet sources.
3. We estimated the jet viewing angles $\Theta$ for the CSOs by using the double-lobe flux ratio. The three CSOs were found to have systematically larger $\Theta$.

4. We plan to observe the CSOs in the future with the VLBI at $5 \mathrm{GHz}$ to measure the expansion speeds of lobes and estimate the ages of these young radio sources.

Acknowledgements. We thank the anonymous referee for valuable comment, and Prof. D.-R. Jiang and Prof. X.-W. Cao for comments on the manuscript. The European VLBI Network is a joint facility of European, Chinese, South African and other radio astronomy institutes funded by their national research councils. This work is supported by the National Natural Science Foundation of China (NNSFC) under grant No. 10773019 and the National Basic Research Program of China (973 Program 2009CB824800).

\section{References}

de Vries, N., Snellen, I. A. G., Schilizzi, R. T., Lehnert, M. D., \& Bremer, M. N. 2007, A\&A, 464, 879

de Vries, N., Snellen, I. A. G., Schilizzi, R. T., Mack, K.-H., \& Kaiser, C. R. 2009, A\&A, 498, 641

Dallacasa, D., Stanghellini, C., Centonza, M., \& Fanti, R. 2000, A\&A, 363, 887

Fanti, C. 2009, AN, 330, 120

Fanti, C., Fanti, R., Dallacasa, D., et al. 1995, A\&A, 302, 317

Giroletti, M., \& Polatidis, A. 2009, AN, 330, 193

Jauncey, D. L., King, E. A., Bignall, H. E., et al. 2003, PASA, 20, 151

Komatsu, E., Dunkley, J., Nolta, M. R., et al. 2009, ApJS, 180, 330

Labiano, A, Barthel, P. D., O’Dea, C. P., de Vries, W. H., \& Baum, S. A. 2007, A\&A, 463, 97

Liu, X., Cui, L., Luo, W.-F., Shi, W.-Z., \& Song, H.-G. 2007, A\&A, 470, 97

Liu, X., Song, H.-G., \& Cui, L. 2009, AN, 330, 145

Luo, W.-F., Yang, J., Cui, L., Liu, X., \& Shen, Z.-Q. 2007, ChJAA, 7, 611

Murgia, M. 2003, PASA, 20, 19

O'Dea, C. P. 1998, PASP, 110, 493

Orienti, M., \& Dallacasa, D. 2008, A\&A, 487, 885

Orienti, M., Dallacasa, D., Tinti, S., \& Stanghellini, C. 2006, A\&A, 450, 959

Orienti, M., Dallacasa, D., \& Stanghellini, C. 2007, A\&A, 475, 813

Ott, M., Witzel, A., Quirrenbach, A., et al. 1994, A\&A, 284, 331

Snellen, I. A. G., Schilizzi, R. T., Miley, G. K., et al. 2000, MNRAS, 319, 445

Snellen, I. A. G., Lehnert, M. D., Bremer, M. N., \& Schilizzi, R. T. 2002, MNRAS, 337, 981

Snellen, I. A. G., Mack, K.-H., Schilizzi, R. T., \& Tschager, W. 2004, MNRAS, 348,227

Stanghellini, C., O’Dea, C. P., Dallacasa, D., et al. 1998, A\&A, 131, 303

Stanghellini, C., O’Dea, C. P., Dallacasa, D., et al. 2005, A\&A, 443, 891

Taylor, G. B., \& Vermeulen, R. C. 1997, ApJ, 485, L9

Tinti, S., Dallacasa, D., de Zotti, G., Celotti, A., \& Stanghellini, C. 2005, A\&A, 432, 31

Torniainen, I., Tornikoski, M., Lähteenmäki, A., et al. 2007, A\&A, 469, 451

Xiang, L., Reynolds, C., Strom, R. G., \& Dallacasa, D. 2006, A\&A, 454, 729 